\title{
Wireless Read-Out of Optical Fiber Sensors
}

\author{
Niels Neumann ${ }^{1}$, Tobias Schuster ${ }^{1}$, Eric Häntzsche ${ }^{2}$, Andreas Nocke ${ }^{2}$, Dirk Plettemeier ${ }^{1}$ \\ ${ }^{1}$ Chair for RF and Photonics Engineering, TU Dresden, 01062 Dresden, Germany, \\ ${ }^{2}$ Institute of Textile Machinery and High Performance Material Technology, \\ TU Dresden, 01062 Dresden, Germany \\ niels.neumann@tu-dresden.de
}

\begin{abstract}
:
Optical fiber sensors cover a wide area of applications. They have unique advantages such as immunity to electromagnetic interference and biocompatibility. However, there are applications where they currently cannot be used because the required fiber-coupled read-out equipment is too expensive, too bulky or not mobile. Radio-over-Fiber technology being already widely used in communications can help to overcome this drawback and pave the way to numerous novel usage scenarios. Using multiplexing techniques (frequency or code division), many signals can be acquired at the same receiver. One important application scenario for the wireless read-out of optical sensors is within buildings and structures. In this paper, sensors for strain, temperature and moisture are presented, their packaging is discussed and the compatibility to the wireless read-out scheme is demonstrated.
\end{abstract}

Key words: optical fiber sensors, wireless, radio-over-fiber, multi-channel, laser

\section{Introduction}

Optical fiber sensors cover a wide range of applications [1,2]. For monitoring of buildings and structures, strain sensors are essential. Besides electrical strain gauges, also optical solutions can be used. Within the scope of carbon reinforced concrete, fiber based solutions employing fiber bragg gratings (FBG) may be embedded into the reinforcement core. Temperature sensors based on FBG or long period gratings (LPG) can be included in order to provide valuable information for building automation. Furthermore, coating optical fibers with hydrogels can yield data on humidity inside the structure.

Also applications in healthcare (especially in point-of-care scenarios) are very promising. High sensitivity as well as enabling spatial resolution in addition to immunity to corrosion and external electromagnetic fields are key advantages of all optical fiber sensors.

Combining photonic components and systems with wireless RF concepts has drawn growing attention in research and development. Combining the advantages of optical systems (low-loss transmission, outstanding performance, immunity to electromagnetic interference, ...) and the benefits of wireless systems (low-cost mass-market realization, mobility and flexibility) leads to Radio-overFiber approaches where a wireless signal is transported over an optical carrier which can be easily converted back to electrical domain and transmitted wirelessly. In Fiber-to-the-Antenna systems, the optical system is extended as far as to the feeding point of the antenna [3].

\section{Radio-over-Fiber technique}

In communications, Radio-over-Fiber (RoF) is a well-established technology to bridge wireless data over optical fibers. A RoF system as shown in Figure 1 consists of a Central Station (CS), Base Stations (BS) and Mobile Stations (MS).

In contrast to conventional optical networks transmitting baseband data, an RF carrier (or, alternatively, an IF carrier) modulated with the payload data is transmitted. This enables simple and cost-effective base stations but requires more complex central stations.

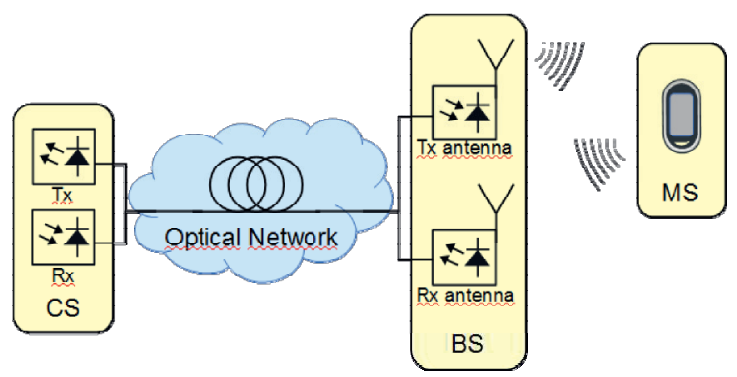

Fig. 1: RoF Communication System

In the CS, RF hardware and digital processing are concentrated. The wireless signal is shaped 


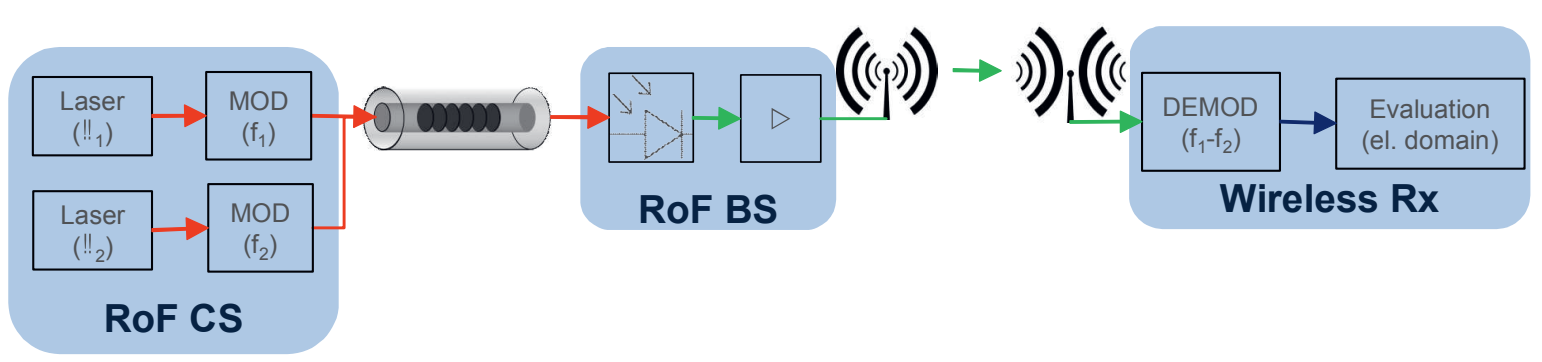

Fig. 2: Wireless Read-Out approach consisting of RoF Central Station, fiber optic sensor, RoF Base station, wireless transmission and wireless receiver where the evaluation is carried out

and modulated onto an optical carrier. This way, long distance transmission in optical networks becomes possible. The BS only consists of a photodiode, an RF power amplifier (if needed) and an antenna. Consequently, simple, cost-effective and maintenance-friendly BS can be realized. Finally, the emitted wireless signal is received by the MS.

\section{Wireless Read-Out approach}

Combining RoF networks with optical sensors facilitates a wireless read-out [4]. Instead of an unmodulated laser and a power measurement or a broadband source and a spectrometer, a RoF signal (optical signal modulated with an RF carrier) is used. This implies replacing the laser source by a RoF CS. The sensor changes this optical signal before it is converted into a wireless signal at the RoF BS. That way, the sensor can be read out by observing the change of the properties of the wireless signal at a wireless receiver at a remote location as depicted in Figure 2. The wireless receiver can be shared among multiple sensors. No complex wiring is needed and cost-effective electronics can be used. This paves the way for new operational areas for fiber optical sensors, e.g. in harsh environment, in a network of numerous sensors or even in mobile applications.

At the RoF CS, a laser with wavelength $\lambda_{1}$ is modulated with the wireless carrier frequency $f_{1}$. Payload data such as sensor ID, status information etc. can be easily added to the wireless signal. There are no restrictions to the wireless signal, i.e. it may use a standard protocol such as IEEE 802.11 WiFi. For calibration purposes (loss in the optical transmission, wireless channel etc.), it is useful to transmit a second signal (laser wavelength $\lambda_{2}$ ) in parallel which will not be altered by the sensor on a second frequency $f_{2}$.

The sensor characteristic changes the optical signal on wavelength $\lambda_{1}$. Consequently, the signal emitted on the wireless frequency $f_{1}$ at the BS will be altered which can be detected at the remote wireless receiver. This principle is illustrated in Figure 3. a)

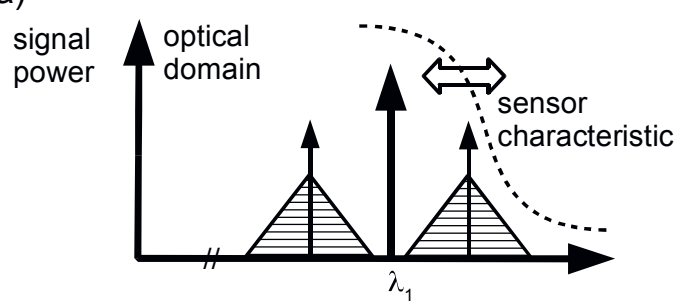

b)

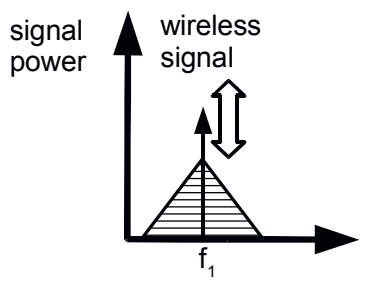

Fig. 3: Scheme of wireless read-out of fiber optic sensor: a) RoF signal changed by sensor in optical domain, b) effect on received wireless signal

\section{Application Scenario and Optical Fiber sensors}

One important application scenario is the monitoring of buildings and critical structures. carbon concrete composite has drawn attention as a promising building material with many advantageous properties. For example, it is possible, to embed textile based sensors in the carbon fiber reinforcement [5].

Interesting quantities within carbon concrete composite are strain, temperature and humidity. While strain measurements are important for structural health monitoring, temperature and humidity sensors are necessary for advanced "smart house" solutions where heating and room climate are actively controlled.

Fiber-based sensors can be realized using gratings, i.e. periodic modulations of the refractive index of the fiber core. These gratings allow to exchange power between different modes that can propagate within the fiber. The specific wavelengths where this power transfer is possible depend on the grating period and can be seen in the transmission spectrum as a minimum. Changing the environmental 
a)

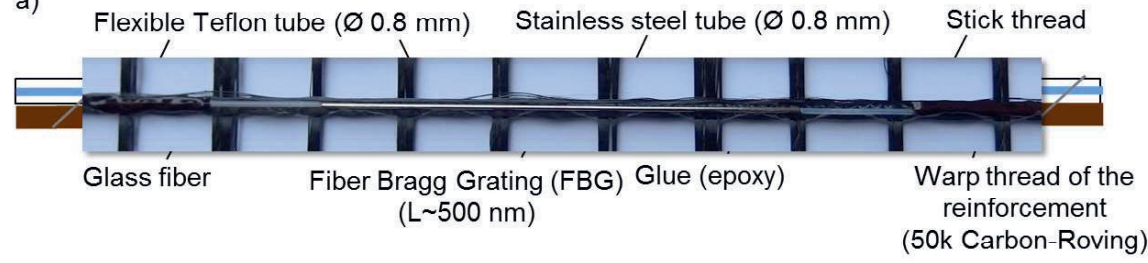

b)

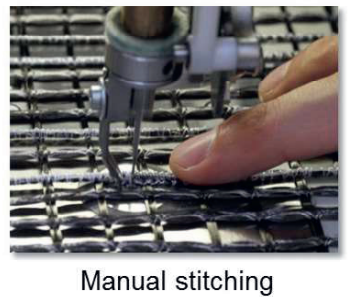

Fig. 4: Optical fiber based strain sensor embedded in carbon reinforcement for carbon concrete composite: a) packaged fiber-based sensor stitched on reinforcement carbon roving, b) stitching process

conditions affects the grating and therefore alters the grating resonance.

Fiber Bragg Gratings (FBG) have grating periods $\Lambda<1 \mu \mathrm{m}$ enabling the coupling of counter-propagating modes. Typically, they are used for strain sensors and temperature sensors. The stretching of the grating under strain changes its period and, consequently, its spectrum. With increasing temperature, the refractive index of the fiber (and also its length) change which has an effect on the grating period and the sensor spectrum.

Long-period Gratings (LPG), on the other hand, couple co-propagating modes and have periods of $\Lambda>50 \mu \mathrm{m}$. They can be used for highly sensitive temperature sensors and humidity sensors. For the latter, the grating is covered with a hydrogel that changes its thickness and refractive index. This way, it changes the grating resonance.

The packaging is a crucial point, here. Many aspects have to be taken into account: First, when tethering the fiber to the carbon fiber reinforcement, the shrinking concrete may lead to small curvatures along the fiber axis. This can damage the fiber and increase the loss (socalled micro-bending loss). Furthermore, the acrylate coating may lose its protective function within the aggressive chemical environment of the concrete. That can cause increased attenuation and lowered lifespan of the fiber due to ingress of moisture. Moreover, a shift of the resonance wavelength of the sensor due to macroscopic bending or mechanical shear force has to be avoided. At the same time, the mechanical properties of the reinforcement should not be impaired and the immersion in the concrete shall not be interfered.

Strain sensors have to be encapsulated in order to ensure a homogeneous strain distribution. For temperature and humidity sensors, crosssensitivity to strain should be minimized. This implies a frictional connection in the case of the strain sensor and force neutral one for the temperature and humidity sensors.
Figure 4 shows the developed package for the strain sensor. Due to the high tensile strength of stainless steel tubes, they are the most suitable option for sensors that are able to measure until the carbon fiber core fails. Additionally, the sensor is shielded from parasitical shearing forces. However, the low flexibility of the stainless steel tubes prevents them from being integrated during the carbon fiber reinforcement production process. Therefore, they are stitched onto the produced carbon fiber reinforcement. By local gluing, a force-fit connection between the packaged sensor and the reinforcement has been achieved.

For the moisture sensor, interaction with the environment in the close vicinity of the sensor is immanent. Therefore, a half open glass tube was chosen as package, see Figure 5a). Glass has a similar thermal expansion coefficient as the fiber which reduces thermally induced cross-sensitivity. It also shields the sensor from parasitic shearing forces. The hydrogel coating on the fiber may interact with the environment. A permeable polyester braid prevents the concrete from directly interacting with the hydrogel, yet allows moisture to enter the sensor area.

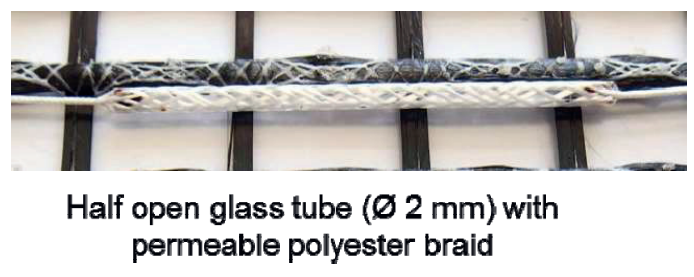

Fig. 5: Packaged optical fiber based moisture sensor embedded in carbon reinforcement for carbon concrete composite

\section{Influence on wireless propagation}

The properties of the wireless propagation through carbon concrete composite has major influence on the wireless read-out of the system. Although the wireless channel is calibrated with a second transmission that does not carry any sensor information, general effects such as attenuation or dispersion may 
affect the maximum transmission distance, achievable sensitivity and resolution.

Steel-reinforced concrete is well researched. However, carbon concrete composite has barely been investigated concerning its RF properties and could behave differently: On the one hand, the conductivity of the carbon fibers is lower than the one of steel. On the other hand, the carbon fiber mesh is much denser than the traditional steel reinforcement. Finally, carbon concrete composite structures typically carry less concrete.

Therefore, the transmission properties of carbon concrete composite have to be assessed in order to provide a comprehensive propagation model. In this paper, the antennas have been placed on top of the concrete and the evaluation has been carried out in the same room so that a simple point-to-point transmission in air could be assumed. This transmission just exhibits free space loss and can be described with the well-known Friis equation.

\section{Measurement results}

First of all, the wavelength dependent behavior of the packaged sensors has been characterized. This is required in order to be able to estimate quantity of interest from the wireless signal after the sensor has influenced the RoF signal as shown in Figure 3.

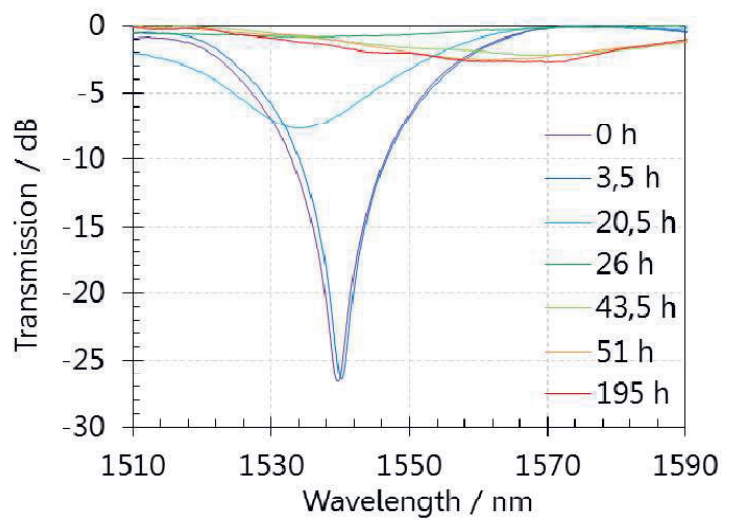

Fig. 6: Shift of grating resonance of the humidity sensor during drying of CF concrete

Figure 6 shows the shift of the grating resonance and its spectral shape during drying of the carbon fiber reinforced concrete. Two influences can be seen: Under heavy moisture, there is a clear grating resonance visible. This resonance is first blue shifted and at the same time the depth of the resonance decreases. Then, under further decrease of the resonance depth, the minimum shifts towards higher wavelengths. The RoF signal remains at one wavelength. At this wavelength, its attenuation decreases during drying and can be assigned to a certain moisture level.

The spectrum of a RoF signal (in this case a DFB laser modulated with a RF signal) is shown in Figure 7a). When the grating spectrum moves, the power of the RoF signal is changed. Consequently, the radiated wireless power depends on the spectral position of the grating which is directly connected with the measured quantity (e.g. moisture, temperature etc.).

The sensitivity of the sensor system therefore not only depends on the sensor (i.e. grating) but also on the RoF signal. Here, the relative position of the RoF signal to the grating is important. Figure 7b) shows the measured sensitivity with respect to the spectral offset between grating minimum and laser line. At the resonance of the grating, the sensitivity is lowest. The slopes of the grating spectrum lead to a high sensitivity. As expected, far away from the grating resonance, the sensitivity converges to zero, again - the behavior of a fiber without grating.

a)

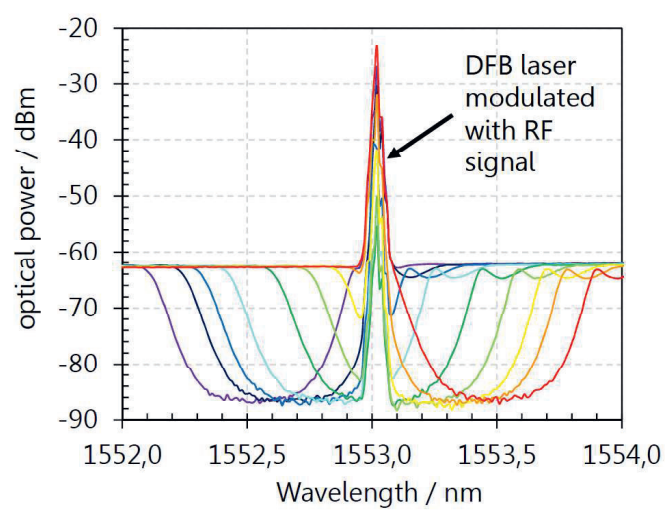

b)

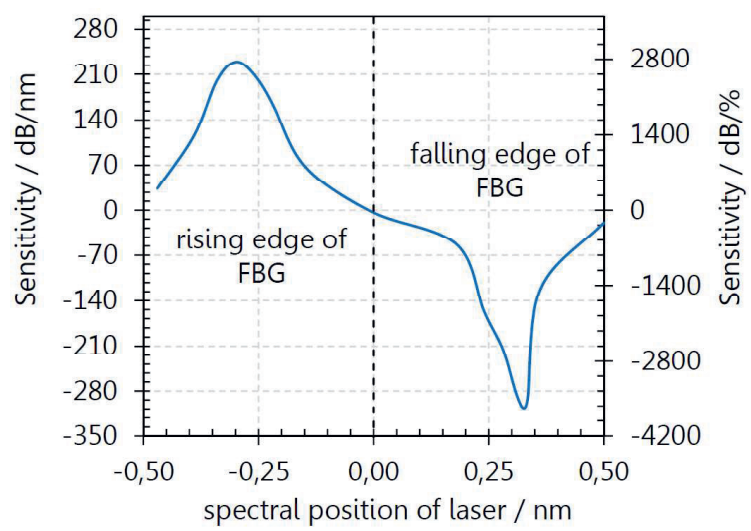

Fig. 7: Wireless read-out of fiber optical strain sensor: a) spectra of DFB laser modulated with RF signal for changing spectral characteristic of the optical fiber sensor, b) sensitivity depending of the spectral position of the laser with respect to the resonance of the optical fiber sensor 


\section{Conclusion}

In this paper, the wireless read-out of optical sensors has been demonstrated using the example of fiber optical sensors embedded in carbon concrete composite.

The underlying Radio-over-Fiber communication scheme has been explained in detail. Also, the strain, humidity and temperature sensors have been introduced. Packaging of the sensors is crucial and was adapted to the respective use case. Finally, a showcase wireless evaluation of a fiber optical sensor has been successfully demonstrated and discussed.

Using RoF signals to link optical sensors with wireless signals has been shown to be feasible and very promising. The areas where optical sensors can be used is widely extended and their evaluation can be simplified.

When the wireless transmission in buildings and structures made from carbon concrete composite involves complicated propagation paths (e.g. through multiple walls), knowing the RF properties of carbon concrete composite is crucial. Therefore, this will be investigated in more detail in the future.

\section{Acknowledgement}

This work has been supported by the German Ministry of Research and Education (BMBF) under the Framework of "C3 - Carbon Concrete Composite" under the Grant Identifiers 03ZZ0305P and 03ZZ0374.

\section{References}

[1] Schuster, T.; Herschel, R.; Neumann, N. \& Schäffer, C. G. Miniaturized Long-Period Fiber Grating Assisted Surface Plasmon Resonance Sensor. Journal of Lightwave Technology, IEEE, 2012, 30, 1003-1008

[2] James, S. W., \& Tatam, R. P. (2003). Optical fibre long-period grating sensors: characteristics and application. Measurement science and technology, 14(5), R49.

[3] Neumann, N.; Trieb, R.; Frach, S. \& Plettemeier, D. FTTA System Demo Using Optical FiberCoupled Active Antennas. Photonics, 2014, 1, 198-210

[4] Neumann, N.; Schuster, T. \& Plettemeier, D. Novel approach for simultaneous wireless transmission and evaluation of optical sensors. Photonics Asia, 2014

[5] E. Häntzsche, A. Nocke, C. Cherif, T. Schuster, N. Neumann, D. Plettemeier, M. Gorges, M. Butler, V. Mechtcherine \& G. Schönfelder: "Multifunktionale Bauteile aus Carbonbeton Integrierte textilbasierte Sensorlösungen zur InSitu- Strukturüberwachung adaptiver Gebäudehüllen", 18. GMA/ITG-Fachtagung Sensoren und Messsysteme 2016 